Acta Crystallographica Section E

Structure Reports

Online

ISSN 1600-5368

Hector Novoa de Armas, ${ }^{a *}$ Bogdan Doboszewski, ${ }^{b}$ Piet Herdewijn $^{b}$ and Norbert Blaton ${ }^{a}$

aLaboratory for Biocrystallography, Faculty of Pharmaceutical Sciences, Katholieke

Universiteit Leuven, Campus Gasthuisberg - O and N2, Herestraat 49, Box 822, 3000 Leuven, Belgium, and ${ }^{\mathbf{b}}$ Laboratory for Medicinal

Chemistry, Faculty of Pharmaceutical Sciences, Katholieke Universiteit Leuven,

Minderbroedersstraat, 3000 Leuven, Belgium

Correspondence e-mail

hector.novoa@pharm.kuleuven.be

Key indicators

Single-crystal X-ray study

$T=293 \mathrm{~K}$

Mean $\sigma(\mathrm{C}-\mathrm{C})=0.004 \AA$

$R$ factor $=0.036$

$w R$ factor $=0.105$

Data-to-parameter ratio $=9.9$

For details of how these key indicators were automatically derived from the article, see http://journals.iucr.org/e.

\section{3-Deoxy-1,2-O-isopropylidene-3-C-methyl- 5-O-(p-tolylsulfonyl)-D-ribofuranose}

In the title compound [alternative name: (2,2,6R-trimethylReceived 23 March 2007 tetrahydrofuro[ $2 R, 3 R-d][1,3]$ dioxol-5S-yl)methyl 4-methylAccepted 14 April 2007 is in the $T$ (twisted) conformation, with atom $\mathrm{C} 3$ exo and atom $\mathrm{C} 4$ endo. The isopropylidene ring is in an envelope conformation. The crystal structure is stabilized by means of van der Waals interactions and weak $\mathrm{C}-\mathrm{H} \cdots \mathrm{O}$ interactions.

\title{
Comment
}

As part of an ongoing research programme in our laboratory, there is a need to obtain all four stereoisomers of 3-hydroxy-2methylbutanoic acid. The $3 R, 2 R$ isomer, (III), can be obtained from 1,2,5,6-di- $O$-isopropylidene-D-glucofuranose via its conversion to the 3-deoxy-3-C-methyl-allo- and -glucocompounds, (I) (Martin et al., 1983; Dang et al., 2000). Both epimers can be separated by chromatography, but this is rather difficult for a larger scale preparation. However, a single stereoisomer at C3, viz. (II), could be easily obtained after degradation of the C5,6 side chain (Robins et al., 2000; Xie et al., 1996), followed by conventional tosylation, as shown in the scheme. In order to assure the correct stereochemistry of the final compound, (III), it is necessary to establish the absolute configuration of the intermediate title compound, (II), in particular the firm confirmation of the configuration at atom C3. Therefore, compound (II) was subjected to an X-ray crystallographic analysis.

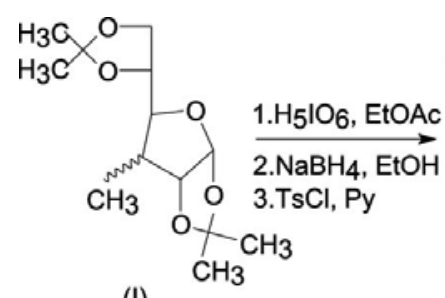

(I)

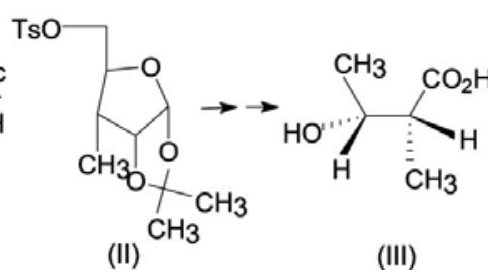

(II)

(III)

In the crystal structure of (II), the puckering parameters (Cremer \& Pople, 1975) for the ribo-pentofuranose ring (O1/ $\mathrm{C} 1 / \mathrm{C} 2 / \mathrm{C} 3 / \mathrm{C} 4)$ are $q_{2}=0.362(3) \AA$ and $\varphi=306.5(4)^{\circ}$, and for the isopropylidene ring $(\mathrm{O} 2 / \mathrm{C} 2 / \mathrm{C} 1 / \mathrm{O} 3 / \mathrm{C} 9), q_{2}=0.253$ (3) $\AA$ and $\varphi=175.7(6)^{\circ}$. The ribo-pentofuranose ring is in the $T$ form (twisted), with atom $\mathrm{C} 3$ exo and atom $\mathrm{C} 4$ endo. The pseudorotation parameters (Rao et al., 1981) for this ring are $P$ $=38.9(3)^{\circ}$ and $\tau_{\mathrm{m}}=38.2(2)^{\circ}$ for the reference bond $\mathrm{C} 2-\mathrm{C} 3$. The configurations at atoms $\mathrm{C} 1, \mathrm{C} 2, \mathrm{C} 3, \mathrm{C} 4$ are $R, R, R, S$, respectively. The $\mathrm{H}$ atoms at $\mathrm{C} 1$ and $\mathrm{C} 2$ are in a bisecting orientation, while those at $\mathrm{C} 3$ and $\mathrm{C} 4$ are in an axial orientation. In this ring, the $\mathrm{O} 1-\mathrm{C} 1$ bond is significantly shorter than the $\mathrm{O} 1-\mathrm{C} 4$ bond, as is found in most 1,2- $\mathrm{O}$-isopropyl- 


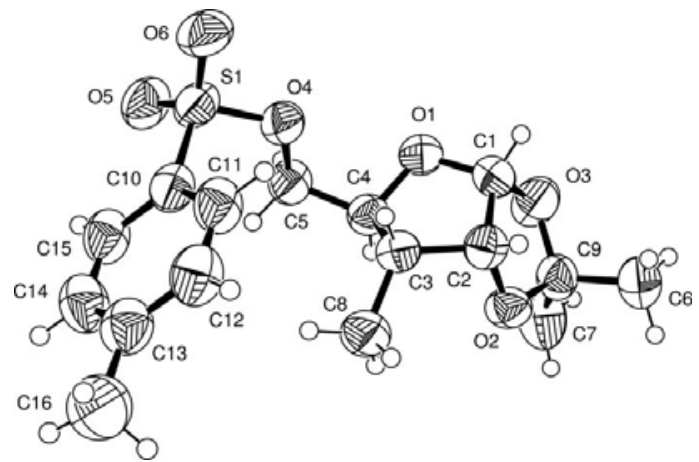

Figure 1

The molecular structure of (II), showing the atom-labelling scheme. Displacement ellipsoids are drawn at the $50 \%$ probability level. $\mathrm{H}$ atoms are represented by circles of arbitrary size.

idene-3,4-disubstituted furanoid rings (Phillips \& Trotter, 1977). The isopropylidene ring conformation can be described as an envelope, with $\mathrm{O} 2$ as the flap atom, with pseudorotation parameters $P=264.1(3)^{\circ}$ and $\tau_{\mathrm{m}}=28.5(1)^{\circ}$ for the reference bond $\mathrm{C} 1-\mathrm{O} 3$.

Since no electron-donor groups are present to form classical hydrogen bonds, the crystal structure of (II) is stabilized by means of weak $\mathrm{C}-\mathrm{H} \cdots \mathrm{O}$ interactions, along with van der Waals interactions.

\section{Experimental}

The details of the synthesis of (II) and its further transformation to the target compound, (III), will be published elsewhere. Compound (II), as a pure ribo isomer, was obtained in 54\% yield starting from (I). Suitable crystals were obtained by slow evaporation of a solution in ethyl acetate (m.p. 393-396 K). Spectroscopic analysis: ${ }^{1} \mathrm{H}$ NMR (300 MHz, $\mathrm{CDCl}_{3}$, $\delta$, p.p.m.): 7.88 ( $d, J=8.2 \mathrm{~Hz}, 2 \mathrm{H}$, aromatic), 7.34 $\left(d, J=8.2 \mathrm{~Hz}, 2 \mathrm{H}\right.$, aromatic), $5.69\left(d, J_{12}=3.5 \mathrm{~Hz}, 1 \mathrm{H}, \mathrm{H} 1\right), 4.52\left(t, J_{12}\right.$ $\left.=J_{23}=4.1 \mathrm{~Hz}, 1 \mathrm{H}, \mathrm{H} 2\right), 4.23\left(d d, J_{45}=2.5 \mathrm{~Hz}, J_{55^{\prime}}=11.1 \mathrm{~Hz}, 1 \mathrm{H}, \mathrm{H} 5\right)$, $4.07\left(d d, J_{5^{\prime} 4}=3.9 \mathrm{~Hz}, J_{5^{\prime} 5}=11.1 \mathrm{~Hz}, 1 \mathrm{H}, \mathrm{H} 5^{\prime}\right), 3.86\left(d t, J_{43}=10.3 \mathrm{~Hz}\right.$, $\left.J_{45}=J_{45^{\prime}}=3.2 \mathrm{~Hz}, 1 \mathrm{H}, \mathrm{H} 4\right), 2.45(s, 3 \mathrm{H}, \mathrm{Me}), 2.02\left(d d q, J_{32}=4.5 \mathrm{~Hz}\right.$, $J_{34}=10.0 \mathrm{~Hz}, J_{3-\mathrm{Me}}=7.5 \mathrm{~Hz}, 1 \mathrm{H}, \mathrm{H} 3$ ), 1.46 and 1.31 (two $s, 3 \mathrm{H}$ each, isopropylidene), $1.03\left(d, J_{\mathrm{Me}-3}=6.8 \mathrm{~Hz}, 3 \mathrm{H}, \mathrm{Me}\right)$. Exact mass calculated for $\mathrm{C}_{16} \mathrm{H}_{22} \mathrm{O}_{6} \mathrm{~S}+\mathrm{Na}=365.10295$; found: 365.10166 .

$\begin{array}{ll}\text { Crystal data } & \\ \mathrm{C}_{16} \mathrm{H}_{22} \mathrm{O}_{6} \mathrm{~S} & V=1713.72(13) \AA^{3} \\ M_{r}=342.40 & Z=4 \\ \text { Orthorhombic, } P 2_{1} 2_{1} 2_{1} & \mathrm{Cu} \mathrm{K \alpha} \text { radiation } \\ a=7.7521(3) \AA & \mu=1.93 \mathrm{~mm}^{-1} \\ b=11.6202(4) \AA & T=293 \mathrm{~K} \\ c=19.0242(11) \AA & 0.61 \times 0.40 \times 0.27 \mathrm{~mm} \\ & \\ \text { Data collection } & \\ \text { Siemens } P 4 \text { four-circle } & 2113 \text { independent reflections } \\ \quad \text { diffractometer } & 2049 \text { reflections with } I>2 \sigma(I) \\ \text { Absorption correction: } \psi \text { scan } & R_{\text {int }}=0.041 \\ \quad \text { (North } \text { et al. }, 1968) \text { and } X E M P & 3 \text { standard reflections } \\ \quad \text { (Siemens, } 1989)] & \text { every } 100 \text { reflections } \\ \quad T_{\text {min }}=0.441, T_{\max }=0.595 & \text { intensity decay: none } \\ 2295 \text { measured reflections } & \end{array}$

\section{Refinement}

$R\left[F^{2}>2 \sigma\left(F^{2}\right)\right]=0.036$

$w R\left(F^{2}\right)=0.105$

$S=1.11$

2113 reflections

213 parameters

$\mathrm{H}$-atom parameters constrained

\author{
$\Delta \rho_{\max }=0.14$ e $\AA_{\circ}^{-3}$ \\ $\Delta \rho_{\min }=-0.26{\mathrm{e} \AA^{-3}}^{-3}$ \\ Absolute structure: Flack (1983), \\ with 210 Friedel pairs \\ Flack parameter: $0.00(2)$
}

Table 1

Selected geometric parameters $\left(\AA{ }^{\circ}{ }^{\circ}\right)$.

\begin{tabular}{lcll}
\hline $\mathrm{S} 1-\mathrm{O} 6$ & $1.421(2)$ & $\mathrm{O} 2-\mathrm{C} 9$ & $1.418(3)$ \\
$\mathrm{S} 1-\mathrm{O} 5$ & $1.422(2)$ & $\mathrm{O} 2-\mathrm{C} 2$ & $1.423(3)$ \\
$\mathrm{S} 1-\mathrm{O} 4$ & $1.5740(19)$ & $\mathrm{O} 3-\mathrm{C} 1$ & $1.400(4)$ \\
$\mathrm{S} 1-\mathrm{C} 10$ & $1.743(3)$ & $\mathrm{O} 3-\mathrm{C} 9$ & $1.420(4)$ \\
$\mathrm{O} 1-\mathrm{C} 1$ & $1.406(3)$ & $\mathrm{O} 4-\mathrm{C} 5$ & $1.456(3)$ \\
$\mathrm{O} 1-\mathrm{C} 4$ & $1.443(3)$ & & \\
$\mathrm{C} 10-\mathrm{S} 1-\mathrm{O} 4-\mathrm{C} 5$ & $73.1(2)$ & $\mathrm{C} 8-\mathrm{C} 3-\mathrm{C} 4-\mathrm{C} 5$ & $76.8(3)$ \\
$\mathrm{S} 1-\mathrm{O} 4-\mathrm{C} 5-\mathrm{C} 4$ & $-153.96(18)$ & & \\
\hline
\end{tabular}

Table 2

Hydrogen-bond geometry $\left(\AA \mathrm{\AA}^{\circ}\right)$.

\begin{tabular}{lllll}
\hline$D-\mathrm{H} \cdots A$ & $D-\mathrm{H}$ & $\mathrm{H} \cdots A$ & $D \cdots A$ & $D-\mathrm{H} \cdots A$ \\
\hline $\mathrm{C} 3-\mathrm{H} 3 \cdots \mathrm{O} 4$ & 0.98 & 2.56 & $2.964(3)$ & 105 \\
$\mathrm{C} 4-\mathrm{H} 4 \cdots 6^{\mathrm{i}}$ & 0.98 & 2.53 & $3.496(3)$ & 168 \\
$\mathrm{C} 15-\mathrm{H} 15 \cdots \mathrm{O}^{2}$ & 0.93 & 2.56 & $2.923(4)$ & 104 \\
$\mathrm{C}^{\mathrm{ii}}-\mathrm{H} 15 \cdots 3^{\mathrm{i}}$ & 0.93 & 2.55 & $3.309(4)$ & 139 \\
\hline
\end{tabular}

Symmetry codes: (i) $x+1, y, z$; (ii) $-x+\frac{3}{2},-y, z+\frac{1}{2}$.

All $\mathrm{H}$ atoms, apart from those of the methyl groups, were placed in geometrically idealized positions and constrained to ride on their parent atoms, with $\mathrm{C}-\mathrm{H}=0.93 \AA$ and $U_{\text {iso }}(\mathrm{H})=1.25 U_{\text {eq }}(\mathrm{C})$. The methyl $\mathrm{H}$ atoms were then constrained to an ideal geometry, with $\mathrm{C}-$ $\mathrm{H}=0.98 \AA$ and $U_{\text {iso }}(\mathrm{H})=1.2 U_{\text {eq }}(\mathrm{C})$, but each group was allowed to rotate freely about its $\mathrm{C}-\mathrm{C}$ bond.

Data collection: XSCANS (Siemens, 1996); cell refinement: $X S C A N S$; data reduction: XSCANS; program(s) used to solve structure: SIR92 (Altomare et al., 1994); program(s) used to refine structure: SHELXL97 (Sheldrick, 1997); molecular graphics: DIAMOND (Bergerhoff, 1996); software used to prepare material for publication: PLATON (Spek, 2003).

HNA is grateful to the Faculty of Pharmaceutical Sciences (Katholieke Universiteit Leuven, Belgium) for funding.

\section{References}

Altomare, A., Cascarano, G., Giacovazzo, C., Guagliardi, A., Burla, M. C., Polidori, G. \& Camalli, M. (1994). J. Appl. Cryst. 27, 435.

Bergerhoff, G. (1996). DIAMOND. Gerhard-Domagk-Strasse 1, 53121 Bonn, Germany.

Cremer, D. \& Pople, J. A. (1975). J. Am. Chem. Soc. 97, 1354-1358.

Dang, H. S., Franchi, P. \& Roberts, B. P. (2000). Chem. Commun. pp. 499-500. Flack, H. D. (1983). Acta Cryst. A39, 876-881.

Martin, O. R., Nabinger, R. C., Ali, Y., Vyas, D. M. \& Szarek, W. A. (1983). Carbohydr. Res. 121, 1983, 302-307.

North, A. C. T., Phillips, D. C. \& Mathews, F. S. (1968). Acta Cryst. A24, 351359.

Phillips, S. E. V. \& Trotter, J. (1977). Acta Cryst. B33, 1003-1007.

Rao, S. T., Westhof, E. \& Sundaralingam, M. (1981). Acta Cryst. A37, 421-425. Robins, M. J., Doboszewski, B., Timoshchuk, V. A. \& Peterson, M. A. (2000). J. Org. Chem. 65, 2939-2945. 
Sheldrick, G. M. (1997). SHELXL97. University of Göttingen, Germany. Siemens (1989). XEMP. Siemens Analytical X-ray Instruments Inc., Madison, Wisconsin, USA.
Siemens (1996). XSCANS User's Manual. Version 2.1. Siemens Analytical

X-ray Instruments Inc., Madison, Wisconsin, USA.

Spek, A. L. (2003). J. Appl. Cryst. 36, 7-13.

Xie, M., Berges, D. A. \& Robins, M. J. (1996). J. Org. Chem. 61, 5178-5179. 